\title{
Commentary Echocardiography and assessing fluid responsiveness: acoustic quantification again into the picture?
}

\author{
Jan Poelaert and Carl Roosens
}

University Hospital Ghent, Department of Intensive Care Medicine and Cardiac Anaesthesia, De Pintelaan, B-9000 Gent, Belgium

Correspondence: Jan Poelaert, jan.poelaert@ugent.be

Published: 23 January 2007

Critical Care 2007, 11:105 (doi:10.1186/cc5140)

This article is online at http://ccforum.com/content/11/1/105

(c) 2007 BioMed Central Ltd

See related research by Cannesson et al., http://ccforum.com/content/10/6/R171

\begin{abstract}
Accurate identification of fluid responsiveness has become an important issue in critically ill patients. Pulse pressure and stroke volume variation have been shown to be reliable predictors of fluid responsiveness. Apart from these two valuable techniques, echoDoppler offers an interesting alternative for estimating the adequacy of filling. Acoustic quantification is a high-tech tool for delineating the blood-tissue interface on-screen in real time. Cannesson and coworkers utilized this technique in ventilated patients to assess stroke area changes, with the intention being to predict fluid responsiveness.
\end{abstract}

Optimization of haemodynamics in critically ill patients necessitates a critical balance between cardiac preload, afterload and myocardial performance. In this context, assessment of fluid responsiveness remains difficult, especially in haemodynamically unstable patients. Many researchers have invested their experience in developing reproducible methods to predict optimal preload safely and adequately. Furthermore, echocardiography may be helpful in estimating fluid responsiveness [1]. Alterations in intrathoracic pressures during mechanical ventilation are known to induce cyclic variations in preloading conditions of the right and left ventricles, resulting in discrete changes in stroke volume and hence in arterial pressure [2]. Significant ventilation-induced undulations in the arterial pressure tracing are clinically related to the presence of hypovolaemia, both in patients with preserved and in those with decreased left ventricular (LV) function [3]. In septic shock patients, analysis of stroke volume variation has been shown to indicate most accurately the presence of fluid responsiveness.

Echocardiography and echo-Doppler have been demonstrated to be an invaluable tool in the haemodynamic management of the critically ill, either through a transthoracic [4] or transoesophageal [5] approach. Estimation of volume status in ventilated intensive care unit (ICU) patients has also been a focus for many ICU echocardiographers. Assessment of the collapsibility of the inferior or superior [6] caval vein is a relatively simple method to identify the presence of fluid responsiveness, although it is strongly influenced by right ventricular function. Echo-Doppler was shown to be a good alternative to pressures obtained by invasive means in demonstrating the presence of fluid responsiveness. In a graded haemorrhagic rabbit model, Slama and coworkers [7] found that ventilation-induced transaortic flow variation could be used in place of invasive pressure variation, underlining the physiological link between pressure and flow [8].

Acoustic quantification (AQ) was introduced in the early 1990s, with the intention being to delineate - both on-screen and in real time - the blood-tissue interface at the level of the various cavities of the heart and great vessels [9]. In the construction of pressure-volume loops, AQ has mostly been exploited as a clever tool to replace the invasive assessment of the volume component [10] in the estimation of endsystolic elastance. Despite such an appealing application of this investigational tool, the transition toward its integration into clinical practice is not so easy. Several drawbacks must be acknowledged, the most important being the fact that the smallest of changes in transoesophageal echo (TOE) probe position relative to the left ventricle can significantly alter estimates of LV contractility. It is therefore more appealing to apply this technology to assessment of cavities that are less mobile (or that can be rendered less mobile). Our research group showed an intriguing correlation between $\mathrm{AQ}$ obtained aortic contour variations and the derived afterload parameters at one side and the Langewouters' model at the other [11].

In the previous issue of Critical Care, Cannesson and coworkers [1] applied the $\mathrm{AQ}$ technique to assess stroke

$\mathrm{AQ}=$ acoustic quantification; $\mathrm{ICU}=$ intensive care $\mathrm{unit} ; \mathrm{LV}=$ left ventricular; $\mathrm{TOE}=$ transoesophageal echo. 
area changes in real time in mechanically ventilated cardiac surgical patients with preserved LV function, as a representative index of fluid responsiveness. In this interesting study, which included a relatively small number of patients, Cannesson and coworkers nicely circumvented the problems associated with change in TOE probe position with respect to the LV short axis by utilizing passive leg raising (trunk lowering from $45^{\circ}$ to $0^{\circ}$ ).

However, as with previous studies using $A Q$, this investigation identified several issues that should be kept in mind before this technique may be routinely implemented to assess fluid responsiveness. First, despite the careful implementation of passive leg raising, a small shift or displacement of the TOE probes in relation to the LV short axis could distort the findings significantly. The analysis of endocardial motion can be confounded if it is applied to patients with segmental wall motion abnormalities [12]. Second, inherently linked to the structure of the myocardial muscle fibres, LV rotations and torsions could be present, which again could interfere with AQ-based assessment. Third, in a subset of coronary patients, a transient increase in load by leg elevation results in a decrease in $\mathrm{dP} / \mathrm{dtmax}$ and a major increase in LV end-diastolic pressure. This impairment is linked to deficient length-dependent regulation of myocardial function [13]. Finally, echo-Doppler provides discontinuous information, which is in contrast to routinely available haemodynamic monitors.

De Backer and coworkers [14] showed that pulse pressure variation was a reliable predictor of fluid responsiveness only if tidal volume was more than $8 \mathrm{ml} / \mathrm{kg}$. Further investigation is necessary to reveal whether the observations reported by Cannesson and coworkers [1] are also valid in patients ventilated with tidal volumes of less than $8 \mathrm{ml} / \mathrm{kg}$ and a higher level of positive end-expiratory pressure (as is the case for patients with acute respiratory distress syndrome). Although dynamic indices of fluid responsiveness are evidently superior to static markers, Doppler echocardiography provides a variety of bedside solutions to problems relating to hypovolaemia in a relatively noninvasive and dynamic manner. The great strength of this tool lies in its power to add supplementary information regarding contractility and afterloading conditions in a direct, comprehensible and physiological manner at the bedside.

\section{Competing interests}

The author(s) declare that they have no competing interests.

\section{Acknowledgements}

Work conducted by the authors is supported by an unrestricted grant from the International Research Centre of the Ghent University, Gent, Belgium.

\section{References}

1. Cannesson M, Slieker J, Desebbe O, Farhat F, Bastien O, Lehot $\mathrm{JJ}$ : Prediction of fluid responsiveness using respiratory varia- tions in left ventricular stroke area by transesophageal echocardiographic automated border detection in mechanically ventilated patients. Crit Care 2006, 10:R171.

2. Perel A, Pizov R, Cotev S: Systolic blood pressure variation is a sensitive indicator of hypovolaemia in ventilated dogs subjected to graded hemorrhage. Anesthesiology 1987, 67:498502.

3. Reuter DA, Kirchner A, Felbinger TW, Weis FC, Kilger E, Lamm P, Goetz AE: Usefulness of left ventricular stroke volume variation to assess fluid responsiveness in patients with reduced cardiac function. Crit Care Med 2003, 31:1399-1404.

4. Vignon P, Mentec H, Terré S, Gastinne H, Guéret P, Lemaire F: Diagnostic accuracy and therapeutic impact of transthoracic and transesophageal echocardiography in mechanically ventilated patients in the ICU. Chest 1994, 106:1829-1834.

5. Poelaert JI, Trouerbach J, De Buyzere M, Everaert J, Colardyn FA: Evaluation of transesophageal echocardiography as a diagnostic and therapeutic aid in a critical care setting. Chest 1995, 107:774-779.

6. Vieillard-Baron A, Chergui K, Rabiller A, Peyrouset O, Page B, Beauchet $A$, Jardin $F$ : Superior vena caval collapsibility as a gauge of volume status in ventilated septic patients. Intensive Care Med 2004, 30:1734-1739.

7. Slama M, Masson H, Teboul JL, Arnout ML, Susic D, Frohlich E, Andrejak M: Respiratory variations of aortic VTI: a new index of hypovolemia and fluid responsiveness. Am J Physiol Heart Circ Physiol 2002, 283:H1729-H1733.

8. Poelaert Jl, Schupfer G: Hemodynamic monitoring utilizing transesophageal echocardiography: the relationships among pressure, flow, and function. Chest 2005, 127:379-390.

9. Cahalan M, lonescu P, Melton H, Adler S, Kee L, Schiller N: Automated real-time analysis of intraoperative transesophageal echocardiograms. Anesthesiology 1993, 78:477-484.

10. Gorcsan J, Gasior T, Mandarino W, Deneault L, Hattler B, Pinsky $M$ : On-line estimation of changes in left ventricular stroke volume by transesophageal echocardiographic automated border detection in patients undergoing coronary artery bypass grafting. Am J Cardiol 1993, 72:721-727.

11. Heerman JR, Segers P, Roosens CD, Gasthuys F, Verdonck PR Poelaert J: Echocardiographic assessment of aortic elastic properties with automated border detection in an ICU: in vivo application of the arctangent Langewouters model. $\mathrm{Am} J$ Physiol Heart Circ Physiol 2005, 288:H2504-H2511.

12. Lang RM, Vignon P, Weinert L, Bednarz J, Korcarz C, Sandelski J, Koch R, Prater D, Mor-Avi V: Echocardiographic quantification of regional left ventricular wall motion with color kinesis. Circulation 1996, 93:1877-1885.

13. De Hert $S$, Vander Linden $P$, Ten Broecke $P$, De Mulder P, Rodrigus I, Adriaensen $\mathrm{H}$ : Assessment of length-dependent regulation of myocardial function in coronary surgery patients using transmitral flow velocity patterns. Anesthesiology 2000, 93: 374-381.

14. De Backer D, Heenen S, Piagnerelli M, Koch M, Vincent JL: Pulse pressure variations to predict fluid responsiveness: influence of tidal volume. Intensive Care Med 2005, 31:517-523. 